\title{
A STUdY ON WEED DisCRIMINATION THROUGH WAVELET TRANSFORM, TEXTURE FEATURE EXTRACTION AND ClassificATION
}

\author{
D.Ashok Kumar ${ }^{1}$ and P.Prema ${ }^{2}$ \\ ${ }^{1}$ Government Arts College, Trichy, TamilNadu, India. \\ ${ }^{2}$ Agricultural College and Research Institute, Madurai-625104. TamilNadu, India
}

\begin{abstract}
Texture based weed classification has played an important role in agricultural applications. In the recent years weed classification based on wavelet transform is an effective method. But the feature extraction is main issue for proper classification of weed species. In this paper, the issue of statistical and texture classification based on wavelet transform has been analysed. The efficient texture feature extraction methods are developed for weed discrimination. Three group feature vector can be constructed by the mean and standard deviation of the wavelet statistical features (WSF), Texture feature as Contrast, Cluster Shade, Cluster Prominence and Local Homogeneity (WCSPH) and Energy, Correlation, Cluster Shade, Cluster Prominence and Entropy features (WECSPE) which are derived from the sub-bands of the wavelet decomposition and are used for classification. Experimental results show that Rbio33 Wavelet with WECSPE texture feature obtaining high degree of success rate in classification.
\end{abstract}

\section{KEYWORDS}

Pre-processing, Wavelets, Texture Features, Neural Network.

\section{INTRODUCTION}

Unwanted plants growing in a place at the wrong time and wrong place in the field can be defined as Weed. The weeds compete with crop for nutrient, water, light and space". Controlling of weed in the agricultural field is more expensive and time-consuming activities. Weed control is a necessary management practice for all cropping systems. Weeds are controlled by manual removal by labourer and applying herbicide uniformly within the field.

Actually the weed infestation rates vary from low to high densities. Based on the weed infection some dosage of herbicide can be applied in the particular location an position.

Mostly all existing weed detection methods the image has been processed in two steps. In the first step, segmentation of vegetation against the background (soil and/or harvest residues) and in second step, detection of the vegetation pixels that represent weeds.

The vegetation can be extracted by combination of color planes such as HIS, RGB and YCbCr method. Green vegetation segmentation can be achieved using vegetative indices techniques such as excess green index (ExG), [1,2] the excess red index (ExR), [3], the excess green minus excess red index (ExGR), Offset Excess Green(OEG)[4], vegetative index (VEG) [5],HIS color model combined with classification methods such as Bayes networks and clustering [6]. The author [7] has explained several vegetation segmentation methods for greenness extraction. 
Our approach Global Histogram Equalization with excess green index (ExG) pre-processing techniques was designed to cope with the variability of natural daylight illumination. The vegetation pixels have been identified, color, position, shape, texture features of weed has extracted for classification. These features are depends on the way the photographic images are taken and the weed species involved. Development of feasible site-specific weed control technology makes the above property.

Wavelet transform is widely used in machine vision in an image processing technique for object detection and classification. For real-time specific weed leaf discrimination that is employing haar wavelet decomposition[8] and 200 highest coefficients was extracted, that is used to classify the broad and narrow weed leaves using a classifier k-Nearest Neighbor (k-NN) and classification accuracy is $94 \%$ for broad weed and $92 \%$ for narrow weed and $97 \%$ for little or no weed. The overall accuracy for weed discrimination is $94.3 \%$.

The author [9] reported that Symlet wavelet transforms along with stepwise linear discriminant analysis (SWLDA) and support vector machines (SVMs) for crop and weed classification for real time selective herbicides systems. Classification accuracy obtained using the symlet wavelet family was $98.1 \%$. To recognize radish and weeds from the field an artificial neural network (ANN) was used and the successful recognition rate in the farms was $93.3 \%$ for radish and $93.8 \%$ for weeds [10].

For automatic detection is based on machine vision or spectroscopy [11]. Geometrical, textural or other statistical features are used to detect the weeds [3] particularly in machine vision methods shape features can be used to discriminate between corn and weeds. Other features such as mean of color information $[12,13]$ and non-morphological analysis based on the texture of the image [13] are used for weed discrimination. For texture analysis [4] wavelet transform provide better multi resolution analytical tool. Higher accuracy rate can be achieved in weed classification.

Texture image feature provides vital characteristics for surface and object identification from image[14]. Major component of image processing is texture analysis and texture features are used in many applications such as remote sensing, quality inspection, medical imaging, etc. So the in this paper texture features are used for weed discrimination. Compared to other features color and shape, wavelet based textures feature provide high degree of accuracy in weed discrimination for site specific real-time for site-specific spraying of infested areas [15].

Our objective of the weed discrimination study was to develop weed discrimination image processing algorithms that are based on texture feature of the image to detect broad and narrow leafed weed with high accuracy without any prior assumptions about environmental factors such as lighting conditions. Our proposed pre-processing technique global histogram equalization method is used to normalize the histograms of the images to illuminate the lighting effect. The vegetation extraction method Excess Green method (2G-R-B) is used to enhance the green pixels of the image. After pre-processing, segmentation techniques have applied to retrieve the weed portion of the image. The retrieved weed RGB image was given as input to the wavelet transform. We evaluated and compared four different wavelet families such as symlet, Daubechies , biorthogonal and reverse biorthogonal wavelet family for weed classification. Using Gray level co-occurrence matrix (GLCM) meaningful statistical and texture features are extracted from the sub-band images and Neural Network techniques are used for classification. Our proposed segmentation method and the reverse biorthogonal 3.3 wavelet families with texture features such as Energy, Correlation, Cluster Shade, Cluster Prominence and Entropy (WECSPE) gave the lesser execution time $19.20 \mathrm{~ms}$ and higher accuracy of $98.9 \%$ for weed discrimination from the agricultural field. 
International Journal of Computer Science \& Information Technology (IJCSIT) Vol 7, No 3, June 2015

\section{MATERIALS AND METHODS}

\subsection{Image Acquisition}

The images used for this study belongs to broad and narrow leaf weed. All the weed images were captured using Nikon digital camera under various lighting conditions (sunny,cloudy)in the field of Agricultural college and Research institute, Madurai. The RGB color images were captured under perspective projection and stored in the JPG format. Figure 1 shows the sample images of a Broadleaves and Narrow weed.

Figure 1: Input images for weed
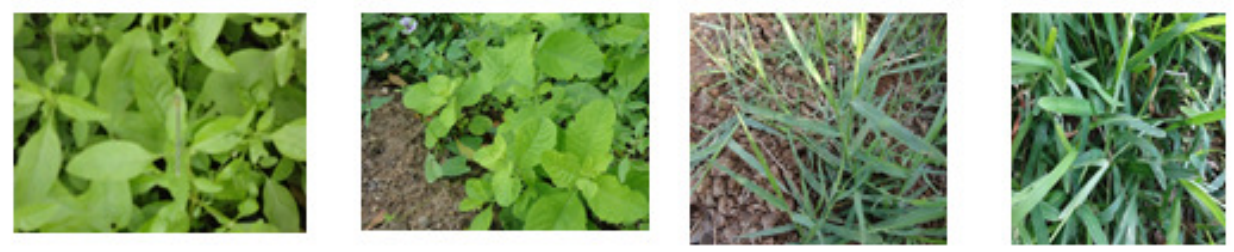

\subsection{Pre-processing}

The initial goal of the weed detection task was to divide the different pixels of the images into two classes as background and plant. Most of the input images have additional parameters such as lighting effects, background information and unnecessary details that may cause misclassification. Hence it is important to remove unnecessary parameters for fast processing and to improve the quality of the image. The proposed pre-processing method is represented in the Figure 2.

Figure 2 : Flow chart for Weed and Background Separation

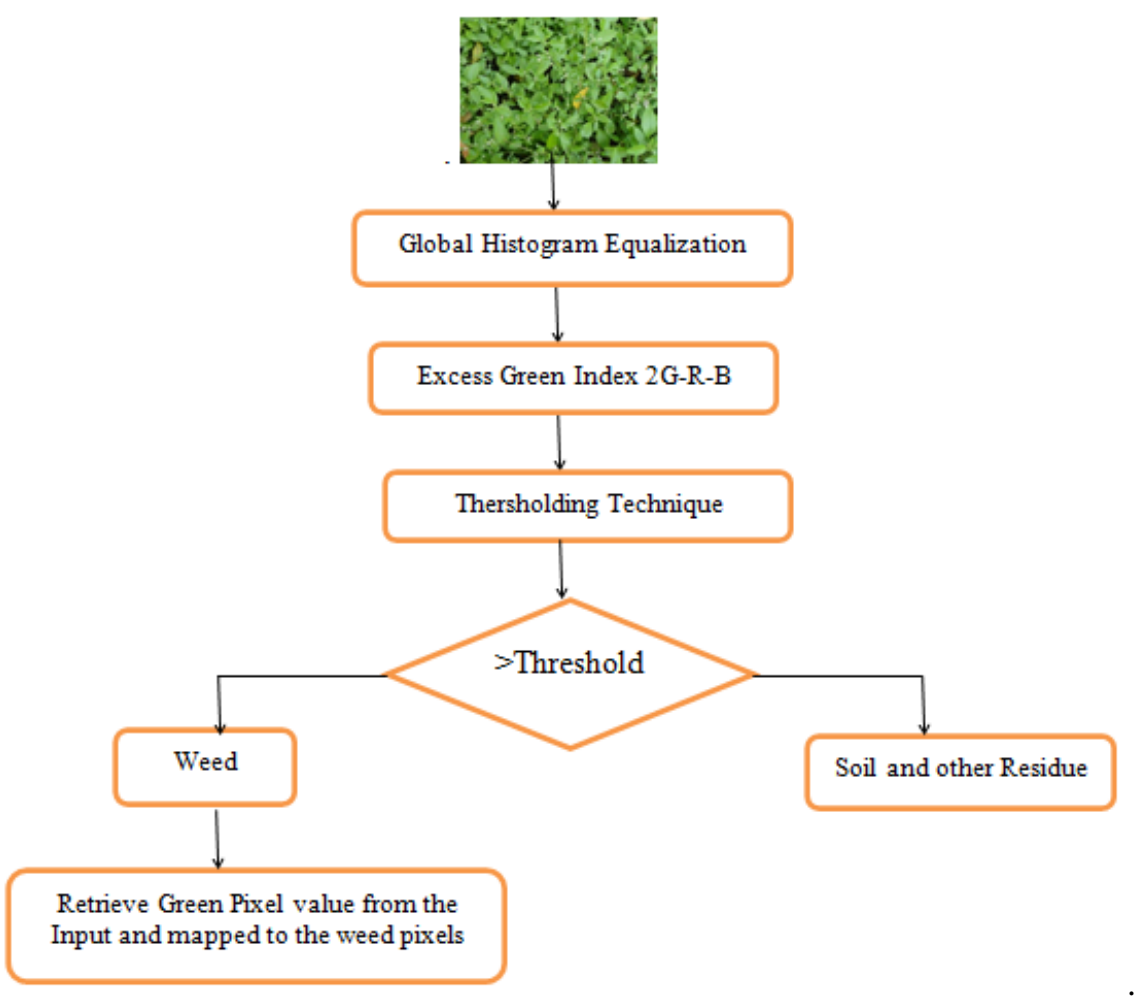


In the pre-processing stage, Global Histogram Equalization (GHE) was used to improve the quality of the image by lengthening the intensity of the dynamic range using the histogram of the whole image. Excess green index is used to extract the greenness of the image. The next goal is to isolate the green parts from the background. For this purpose several thresholding methods were applied. For automatic weed discrimination we computed the average gray level was set as threshold [16]. The binary image can be obtained based on the threshold. The pixel values greater than the threshold are labelled as white, i.e. they contain a high degree of greenness. The outputs of the pre-processing stage are represented in Figure 3.

Figure 3 : Result of Pre-processing techniques
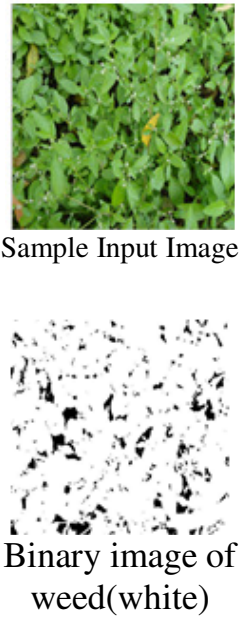

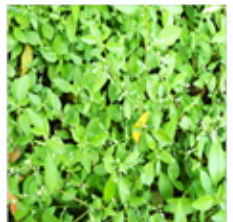

Global Histogram Equalization Image

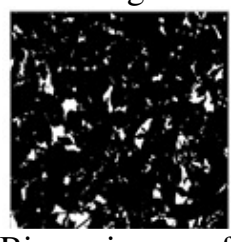

Binary image of

Background(White)

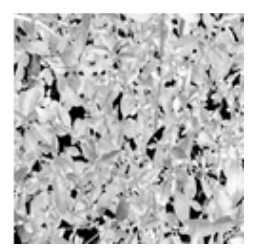

Excess Green output

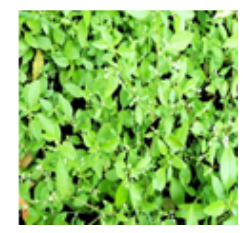

RGB Image for weed

\subsection{Wavelets transform}

Wavelet is a widely used multi-resolution tool in image processing $[4,9,15]$. Wavelet transform is the best method to represent both time and frequency content of the image. There are number of ways to separate the low(smooth variations in color) and the high frequency components(the edges which give details). The input image can be decomposed using discrete wavelet transform. To extend the wavelet transform to two dimensions, the input image is down sample in the horizontal and vertical directions. The output produces four sub bands at each decomposition denoting the horizontal frequency, vertical frequency represented as high-high $(\mathrm{HH})$, high-low (HL), low-high (LH) and low-low (LL) image sub bands. Continuously applying the same scheme to the low-low sub band a multiresolution decomposition can be achieved. Figure 3 shows the normal layout of such decomposition. At each scale the sub bands are sensitive to frequencies and the LH, HL and HH sub bands are sensitive to vertical, horizontal and diagonal frequencies respectively. Figure 4 shows a DWT decomposition of the narrow weed image.

Figure 4: Three level decomposition of Discrete Wavelet Transform

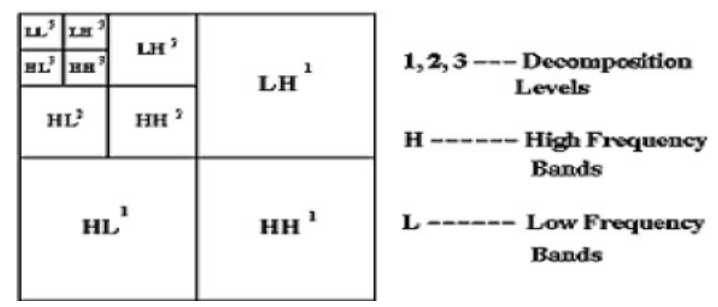


The Input images were decomposed using different types of wavelet families. The 2D RGB image was represented as I, the decomposition of the wavelet transform first level can be expressed as $I=A_{1}+D_{1}$, where $A_{1}$ and $D_{1}$ represent the approximation and details coefficients respectively. In the image is decomposed up to multilevel the Equation can be written as

$$
I=A_{j}+D_{j}+D_{j-1}+D_{j-2}+\ldots . . D_{2}+D_{1}
$$

Where $\mathrm{j}$ represents number of wavelet decomposition. For feature extraction, only approximation coefficients are used, the detail coefficients consist of noise. Each image was decomposed up to three levels $j=3$. Figure 5 shows the result of applying wavelet transform (Reverse Biorthogonal3.3) up to three levels on a sample image. In this study three different level of decomposition based on the four different wavelet function namely Daubechies(db4,db10),Symlets(sym4,sym8),Biorthogonal(bior3.3,bior6.8), Reverse Biorthogonal (rbio3.3,rbio6.8) were used.

Figure 5 : Reverse Biorthogonal3.3 Wavelet Transform (a). Three level wavelet decomposition (b) Reconstructed image

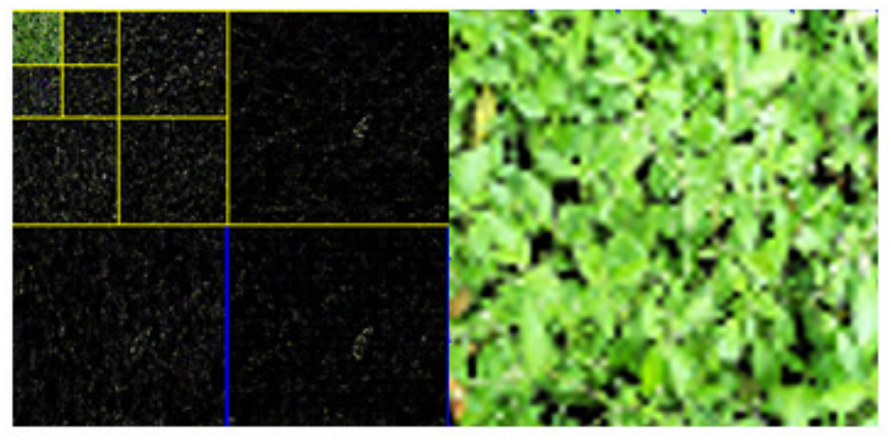

\subsection{Feature Extraction}

Texture is defined as an entity having group of mutually related pixels within an image. This group of pixels is represented as texture elements $[4,18]$.The aim of the weed discrimination analysis was to classify the objects derived by image segmentation into defined number of classes according to their specific features. The wavelet energy texture features were efficiently used for texture classification and segmentation. First order statistical features mean, standard deviation and second order statistical features like cluster shade and cluster prominence are calculated from GLCM and used for classification of broad and narrow leaf weed [3].Five texture features such as Energy, Entropy, Inertia and local homogeneity were extracted from coefficient of Discrete wavelet transform are used texture classification of crop and weed. In real time automatic spray control system [17], hundred highest average feature coefficients were selected from each level of decomposition and Euclidian distance method was used to recognize and differentiate broad and narrow weed. Sum of all the approximation coefficients at each level of decomposition are considered as feature vector for classification of Broad and Narrow leaf weed [9].

The proposed work introduces a new texture based feature extraction method for weed discrimination called Multi Resolution Combined Statistical and Texture Feature (MRCST). MRCST is a combination of first order, second order statistical and texture properties. Using GLCM [17] number of texture features are extracted from the input image and stored in a database for weed discrimination. Texture classification involves learning and classification. In the learning phase, the original image is decomposed using Discrete Wavelet Transform (DWT) with three level of decomposition of different wavelet family. Features such as mean $\mu$ and standard deviation $\sigma$ are calculated from each of these wavelet sub-bands and are used to 
construct a global feature vectors as Wavelet Mean Standard deviation $\mathrm{G}=[\mathrm{G} \mu, \mathrm{G} \sigma]$, where $\mathrm{G} \mu=\left[\mu_{1}\right.$ , $\left.\mu_{2}, \ldots . \mu_{\mathrm{S}}\right], \mathrm{G} \sigma=\left[\sigma_{1}, \sigma_{2}, \sigma_{\mathrm{S}}\right]$, From the GLCM texture feature as Contrast ,Cluster Shade, Cluster Prominence and Local Homogeneity (WCSPH) and Energy , Correlation ,Cluster Shade, Cluster Prominence and Entropy features (WECSPE) and stored in the Features set for the purpose of weed classification.

We use the following notation: $G$ is the number of gray levels used. $P(i, j)$ is the Grey level cooccurrence matrix. $\mu$ is the mean value of P. $\mu x, \mu y$ are the means of Px and Py. Px(i) is the $i^{\text {th }}$ entry in the marginal-probability matrix obtained by summing the rows of $P(i, j)$ :

$$
\begin{aligned}
& P_{x}(i)=\sum_{j=0}^{G-1} P(i, j) \\
& P_{y}(j)=\sum_{i=0}^{G-1} P(i, j) \\
& \mu_{x}=\sum_{i=0}^{G-1} i \sum_{j=0}^{G-1} P(i, j)=\sum_{i=0}^{G-1} i P_{x}(i) \\
& \mu_{y}=\sum_{i=0}^{G-1} \sum_{j=0}^{G-1} j P(i, j)=\sum_{j=0}^{G-1} j P_{y}(j)
\end{aligned}
$$

Four Texture features were extracted from coefficient of DWT. The texture features of Contrast, Cluster shade, Cluster Prominence and Local Homogeneity are calculated using the following formula

$$
\begin{aligned}
& \text { Entropy }=-\sum_{i=1}^{M} \sum_{j=1}^{N} P[i, j] \log P[i, j] \\
& \text { Energy }=\sum_{i=1}^{M} \sum_{j=1}^{N} P^{2}[i, j] \\
& \text { CONTRAST }=\sum_{n=0}^{G-1} n^{2}\left\{\sum_{i=1}^{G} \sum_{j=1}^{G} P(i, j)\right\}, \quad|i-j|=n \\
& \text { SHADE }=\sum_{i=0}^{G-1} \sum_{j=0}^{G-1}\left\{i+j-\mu_{x}-\mu_{y}\right\}^{3} \times P(i, j) \\
& \text { PROM }=\sum_{i=0}^{G-1} \sum_{j=0}^{G-1}\left\{i+j-\mu_{x}-\mu_{y}\right\}^{4} \times P(i, j)
\end{aligned}
$$

\subsection{Feature Classification}

Various classification techniques are used for weed discrimination and disease identification. Most of the classification is based on population variance [5], weed coverage rate (2D-WCR) [6], histogram analysis [7]. All the above methods, the value of image pixels are summed up and set the threshold value for weed image classification. This may cause misclassification of real time weed images, because there are no specific criteria for selecting a threshold value. To overcome the above problem automated classification methods have also been used. The classification methods are support vector machines (SVMs) [17], and neural networks (NNs) and active shape model [19] used for weed discrimination and seed identification. In this paper Back propagation Neural Network was used to classify the input weed as broad and narrow leaf weed. Figure 6 represent the texture feature extraction and classification process. 
Fig 6 : Process flow of texture feature extraction and classification

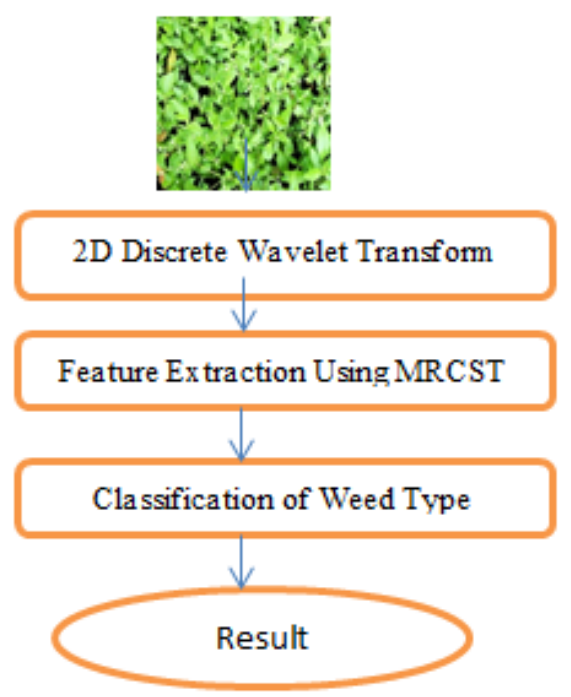

\subsection{Neural Network}

For weed recognition and classification a back propagation neural network was used. The node or elements are composed, which is operating in parallel. These elements are stimulated by biological nervous systems. The network function is determined largely by the connections between elements. A neural network can be trained to perform a particular function by adjusting the values of the connections (weights) between elements. The network is adjusted, based on a comparison of the output and the target, until the network output matches the target image. Feature of 50 images of broad and narrow leaf weeds were used to build ANN model. Set of Feature values are assigned to the input layer of neural network. The number hidden layer will be selected based on the following formula.

No. of hidden layer $=($ number of attribute + number of classes $) / 2$.

The expected output in the training file was $\{1\}$ for Broad weed and $\{0\}$ for narrow weed.

\subsection{Result and Discussion}

Multilevel wavelet decomposition is a technique used in weed discrimination. In this crop/weed discrimination technique is based on wavelet transforms comprised of Daubechies, Mayer and Biorthogonal and compared it to a Gabor filtering. From this wavelet algorithm comparison Daubechies and Mayer gave the best results $(84.6 \%$ \& $84.1 \%)$ and the Biorthogonal is the worst one. Biorthogonal wavelet gave the result $76.4 \%$ and Gabor filter contributed the result as $73.7 \%$ [15] . For weed classification the author[17] used wavelet based algorithm as Symlets, Daubechies, Biorthogonal and Reverse Biorthogonal. Among those the Biorthogonal wavelet with energy features gave the best result (97.64\%).

In this paper the proposed algorithm classified real-time site specific weed discrimination based on wavelet families Daubechies(db4,db10), Symlets(sym4,sym8), and Reverse Biorthogonal (rbio3.3,rbio6.8) with three group of texture features as Mean and standard deviation, texture feature as Contrast ,Cluster Shade, Cluster Prominence and Local Homogeneity (WCSPH) and Energy , Correlation ,Cluster Shade, Cluster Prominence and Entropy features (WECSPE). To build the database 50 images are used for each class (broad and narrow weed. Three levels of decomposition are applied to all the type of wavelet families. And three group of features 
sets(MWS, WCSPH, WECSPE) are extracted from approximation and details coefficients of from different wavelet families and all the decomposition level and are stored. All the coefficients (detail and approximation) contains some useful information, it will necessary requires for proper classification and higher accuracy. Feature sets are assigned as input to the Neural Network. Number of Hidden layer is chosen based on the number of inputs. Neural network techniques were applied separately to all the three features set of different wavelet families. Confusion matrix is used to find the Broad and narrow weed classification accuracy.

Figure 7 shows the level wise execution time for different wavelet family. The Average elapse time for different wavelet family up to three level of decomposition represented in Table 1. From the Table 1 , level 1 execution time for all the wavelet family is high compared to the level 2 and level3.The level1 execution time for Reverse Biorthogonal 3.3 wavelet is $55.88 \mathrm{~ms}$ and Symlet8 family is $56.82 \mathrm{~ms}$, Daubechies4 wavelet is $58.16 \mathrm{~ms}$ and Biorthogonal 6.8 wavelet is $63.02 \mathrm{~ms}$. In level2 the execution for Reverse Biorthogonal3.3 wavelet is $14.60 \mathrm{~ms}$ and Symlet8 family is $13.18 \mathrm{~ms}$, Daubechies4 wavelet is $11.32 \mathrm{~ms}$ and Biorthogonal 6.8 wavelet is $15.77 \mathrm{~ms}$. The elapsed time for level3 for the above wavelet family is $6.30 \mathrm{~ms}, 6.85 \mathrm{~ms}, 5.84 \mathrm{~ms}$ and $6.20 \mathrm{~ms}$.

Figure 7: Level wise Average processing time (s) for wavelet algorithm

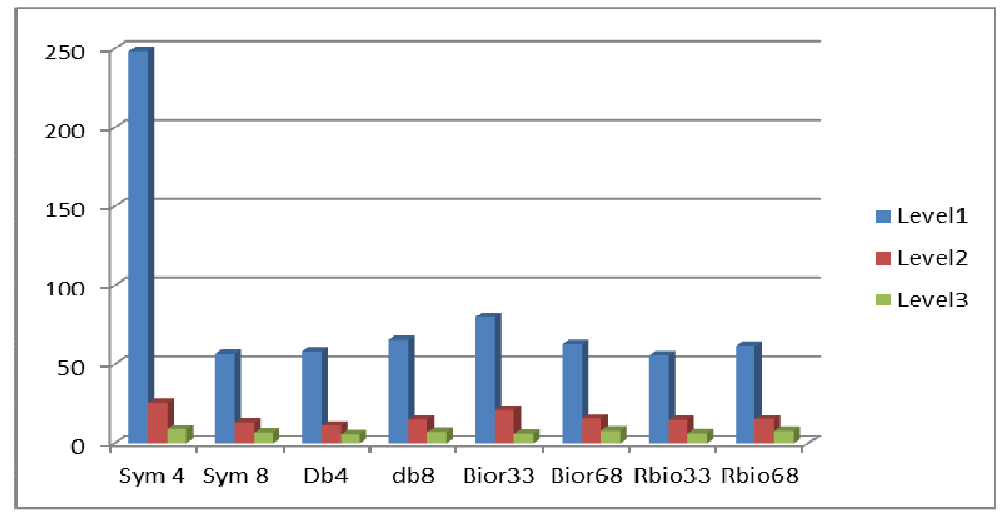

From Table 1 and Figure 8 shows the elapsed time for three level wavelet decomposition of weed discrimination. In Daubechies (Db4) wavelet the elapsed time is $18.83 \mathrm{~ms}$ and Reverse Biorthogonal (rbio3.3) wavelet is $19.20 \mathrm{~ms}$ which is less compared to the other wavelet transform.Symlet 4 has an highest elapsed time as $70.75 \mathrm{~ms}$ and Biorthogonal wavelet as $26.85 \mathrm{~ms}$. The elapsed time for three level decomposition for Biorthogonal wavelet6.8 is $26.85 \mathrm{~ms}$ and Reverse biorthogonal6.8 is $21.27 \mathrm{~ms}$.Compare to all the wavelet Daubechies(Db4) has lowest elapsed time as $18.83 \mathrm{~ms}$.

Table 1: Average Elapsed time for Wavelet Family

\begin{tabular}{|l|c|}
\hline Wavelet Family & Execution Time(ms) \\
\hline Sym4 & 70.75 \\
\hline Sym8 & 19.21 \\
\hline Db4 & 18.83 \\
\hline Db8 & 21.92 \\
\hline Bior33 & 26.85 \\
\hline Bior68 & 21.66 \\
\hline Rbio33 & $\mathbf{1 9 . 2 0}$ \\
\hline Rbio68 & 21.27 \\
\hline
\end{tabular}


International Journal of Computer Science \& Information Technology (IJCSIT) Vol 7, No 3, June 2015

Figure 8 : Execution time for Wavelet Family Decomposition upto three level

Execution Time(ms)

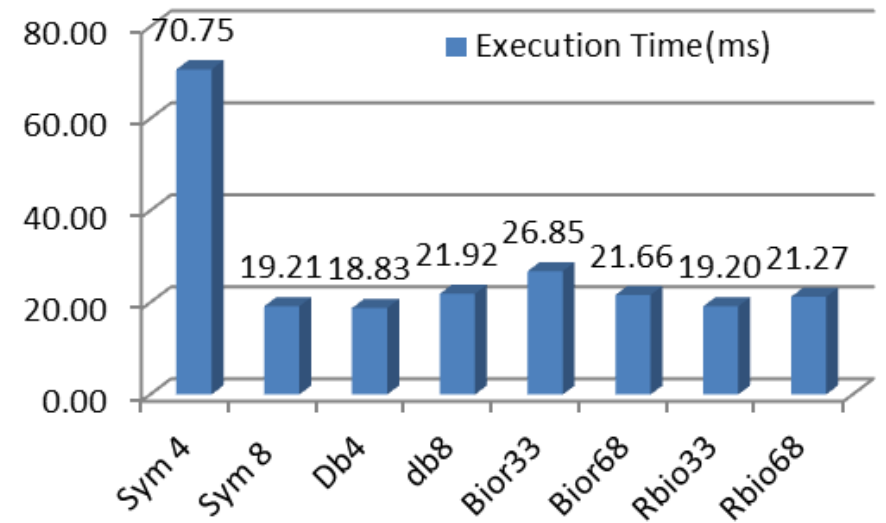

Broad and narrow weed texture feature set for wavelet families are classified with neural network. The Figure 9 and Table 2 show the classification of broad and narrow weeds using different types of wavelets at three levels of decompositions.

Table 2: Accuracy of Wavelet Texture Features

WMS :Wavelet statistical features(WMS), WCSPH : Contrast ,Cluster Shade, Cluster Prominence and Local Homogeneity Texture feature WECSPE : Energy, Correlation, ,Cluster Shade, Cluster Prominence and Entropy features

\begin{tabular}{|l|l|l|l|}
\hline $\begin{array}{l}\text { Wavelet } \\
\text { Family }\end{array}$ & WMS & WCSPH & WESPEnt \\
\hline Sym4 & 76.67 & 91.67 & 96.33 \\
\hline Sym8 & 73.67 & 93.33 & 96.67 \\
\hline Db4 & 77.34 & 95.33 & 96.33 \\
\hline Db10 & 75.67 & 90.42 & 83.33 \\
\hline Bior33 & 76.87 & 90.67 & 96.67 \\
\hline Bior68 & 76.87 & 92.33 & 93.33 \\
\hline Rbio33 & $\mathbf{8 3 . 6 7}$ & $\mathbf{9 8 . 7}$ & $\mathbf{9 8 . 9}$ \\
\hline Rbio68 & 76.67 & 93.33 & 96.67 \\
\hline
\end{tabular}

In Figure 9 the classification accuracy for the texture for Mean and standard deviation for the wavelet family Sym4, Sym8, Db4, Db10, Bior33, Bior68, Rbio33 and Rbio68 are 76.67\%, 73.67\%, 77.34\%,75.67\%,76.87\%,76.87\% ,83.67 and 76.67. The wavelet Reverse Biorthogonal wavelet3.3 wavelet have high accuracy as $83.67 \%$. For the texture feature Contrast ,Cluster Shade, Cluster Prominence and Local Homogeneity (WCSPH) the classification accuracy the wavelet family are represented in Figure 9. Compare to the other wavelet the highest accuracy $86.67 \%$ achieve from the Reverse Biorthogonal3.3. It is obvious form the Table 2 and Figure. 9 that the classification through Reverse Biorthogonal3.3 wavelet (Rbio33) with Energy, Correlation, ,Cluster Shade, Cluster Prominence and Entropy features (WESPEnt) texture features 
is more efficient due to highest accuracy $98.9 \%$ and less execution time for three level of decomposition.

Figure 9: Accuracy of Wavelet Texture Features as WMS, WCSPH and WECSPE

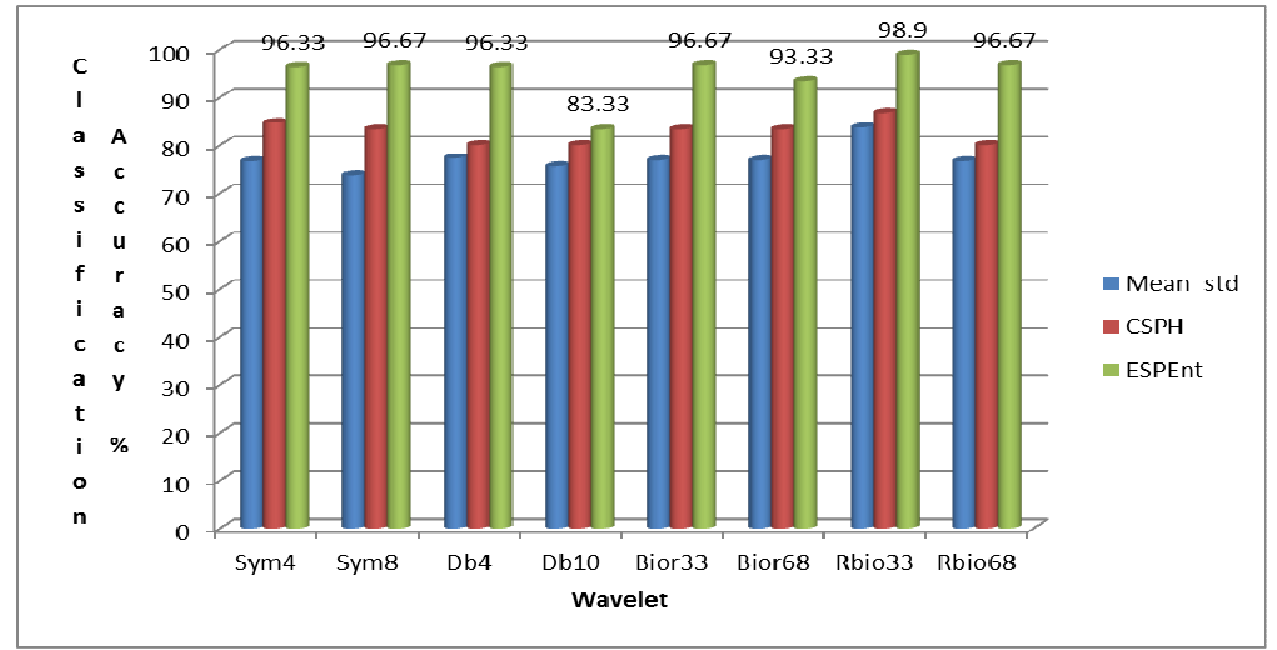

\section{Comparison with Existing Algorithm}

The classification accuracy of Broad and narrow weed was compared to the existing algorithm the author [19] has proposed a real-time system had an accuracy of about $81 \%$ due to the classification based on the highest intensity value taken from the histogram. The author [4] developed a method using Offset Excess Green , Db4 wavelet transform and Multiresolution combined statistical an spatial frequency features for the broad and narrow weed classification. The overall classification accuracy is this method is $98.55 \%$. Using Global Histogram Equalization, Symlet wavelet and approximation coefficient of each level (four level) feature values and SVM classification method, the accuracy of $98.1 \%$ can be obtained [9]. Our proposed algorithm use Global Histogram equalisation, Offset excess green and thresholding techniques are used for segmentation and three level wavelet decomposition of different wavelet families, and three group of texture features are extracted detail and approximation coefficient of three levels and Neural network and confusion matrix are used for classification. From the result of the proposed algorithm Reverse Biorthogonal 3.3 wavelet families with texture features as Energy, Correlation, ,Cluster Shade, Cluster Prominence and Entropy features (WECSPE) gave the accuracy of $98.9 \%$.The results are shown in Fig 10. Compared with the existing algorithm for weed discrimination, our approach gave best results for weed discrimination and useful for site specific herbicide application.

Fig. 10. The Comparison result for proposed algorithm and the existing algorithm

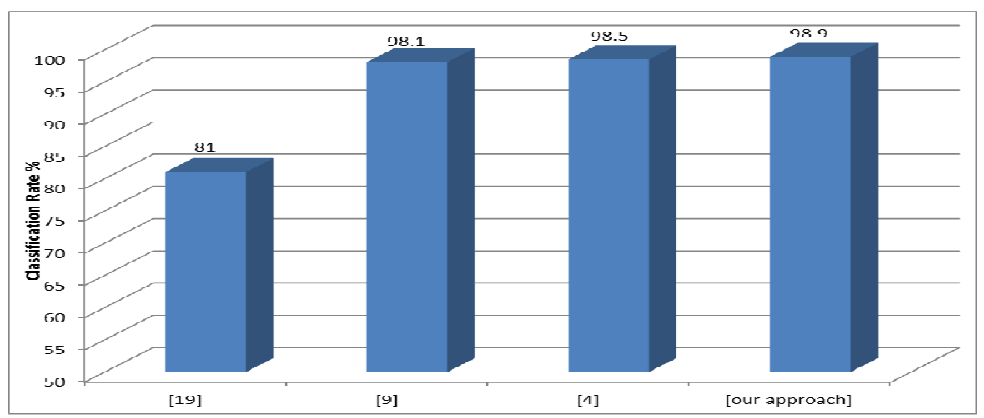


International Journal of Computer Science \& Information Technology (IJCSIT) Vol 7, No 3, June 2015

\section{CONCLUSIONS AND FUTURE SCOPE}

In this study, we proposed an accurate and efficient method for real-time specific weed discrimination using Global Histograms Equalization, Excess Green Index pre-processing techniques and multilevel (three level) wavelet decomposition to extract the prominent texture features from images and Neural Network classification techniques. To increase the accuracy and efficiency, we developed efficient pre-processing methods and feature extraction techniques. We verified four different types of wavelet families Daubechies(db4,db10),Symlets(sym4,sym8) and Reverse Biorthogonal (rbio3.3,rbio6.8) with three group of texture features are extracted and used for Neural Network classification techniques. Our proposed approach using Reverse Biorthogonal (rbio3.3) wavelet family with Energy, Correlation, Cluster Shade, Cluster Prominence and Entropy (WECSPE) features yielded a classification accuracy of $98.9 \%$. Compared to the existing algorithm our proposed algorithm indicates that Rbio3.3 is a promising wavelet due to its high accuracy, less error rate and elapsed time and it is suited for real-time specific weed discrimination. To improve the classification accuracy curvelet can be used.

\section{REFERENCES}

[1] Woebbecke, D., Meyer, G., VonBargen, K., Mortensen, D.,(1995),"Color indices for weed identification under various soil, residue, and lighting conditions", Trans. ASAE, vol 38 (1), pp 271281.

[2] Ribeiro, A., Fernández-Quintanilla, C., Barroso, J., García-Alegre, M.C., (2005).’Development of an image analysis system for estimation of weed", Proceedings $5^{\text {th }}$ European Conf. On Precision Agriculture (5ECPA), pp. 169-174.

[3] Meyer, G.E., von Bargen, K., Woebbecke, D.M., Mortensen, D.A.,(1994),"Shape features for identifying young weeds using image analysis", ASAE paper 94-3019, American Society of Agricultural Engineers, St. Joseph,MI, USA.

[4] R.S. Sabeenian and V. Palanisamy,(2010),"Crop and weed discrimination in agricultural field using MRCSF". Int. J. Signal and Imaging Systems Engineering, Vol. 3, No. 1, pp.61-69.

[5] Hague, T., Tillet, N., Wheeler, H.,(2006)."Automated crop and weed monitoring in widely spaced cereals", Precis. Agr, Vol 7, pp 21-32.

[6] Hemming, J., Rath, T.,(2001),'Computer-vision based weed identification under field conditions using controlled lighting",J. Agr. Eng. Res. Vol 78 (3), pp 233-243.

[7] Meyer, G., Neto, J.,(2008),"Verification of color vegetation indices for automated crop imaging applications",Comput. Electron. Agr. Vol 63, pp 282-293.

[8] Irshad Ahmad, Muhammad Hameed Siddiqi ,Iram Fatima ,Sungyoung Lee, Young-Koo Lee,(2011) "Weed Classification Based on Haar Wavelet Transform via k-Nearest Neighbor (k-NN) for RealTime Automatic Sprayer Control System”,ICUIMC '11, February 21-23, Seoul, Korea.

[9] Muhammad Hameed Siddiqi, Seok-Won Lee And Adil Mehmood Khan,(2014) "Weed Image Classification using Wavelet Transform,Stepwise Linear Discriminant Analysis, and Support Vector Machines for an Automatic Spray Control System", JOURNAL OF INFORMATION SCIENCE AND ENGINEERING, Vol 30, pp 1253-1270.

[10] S. I. Cho; D. S. Lee; J. Y. Jeong, (2002),"Weed-plant Discrimination byMachine Vision and Artificial Neural Network", Biosystems Engineering, Vol. 83 (3), pp 275-280.

[11] Wang, N., Zhang, N., Dowell, F.E., Sun, Y., Peterson, D.E.,(2001),'Design of an optical weed sensor using plant spectral characteristics". Trans. ASAE, Vol. 44 (2), pp 409-419.

[12] Cho, S.I., Lee, D.S., Jeong, J.Y.,(2002),”Weed-plant discrimination by machine vision and artificial neural network",.Biosyst. Eng. Vol 83(3), pp 275-280.

[13] Lamm, R.D., Slaughter, D.C., Giles, D.K.,(2002),'Precision weed control system for cotton",Trans. ASAE, Vol 45 (1),pp 231-238.

[14] Haralick, R.M. (1979) 'Statistical and structural approaches to texture', IEEE Proceedings, Vol. 67, pp.786-804.

[15] J. Bossu, Ch. Gée, G. Jones, F. Truchetet ,(2009),"Wavelet transform to discriminate between crop and weed in perspective agronomic images", computers and electronics in agriculture", Vol 65,pp133-143. 
International Journal of Computer Science \& Information Technology (IJCSIT) Vol 7, No 3, June 2015

[16] M. Guijarro, G. Pajares, I. Riomoros, P.J. Herrera, X.P. Burgos-Artizzue, A. Ribeiroe,(2011),"Automatic segmentation of relevant textures in agricultural images",Computers and Electronics in Agriculture, Vol 75,pp 75-83.

[17] Siddiqi, M.H.,S.B.T Sulaiman,I,Faye and I.Ahmed(2009), "A Real Time Specific Weed Discrimination System using Multi-Level Wavelet Decomposition”, International Journal of Agricultural and Biology, Vol 11, pp 559-565.

[18] K.K.Ghazali ,M.F.Mansor,M.M.Mustafa and A.Hussain,(2007),"Feature Extraction Techniques using Discrete Wavelet transform for Image classification", IEEE $5^{\text {th }}$ Student conference on Research and Development.

[19] C.Anucti and P.PBekaert," SIFT-CCH:Increasing the SIFT distinctness by color co-occurrence histogram" in proceeding of the 5th International symposium on Image and Signal Processing and Analysis, 2007,pp.130-135.

\section{Authors}

P.Prema received her B.Sc(Computer Science) degree in V.V.Vanniya Perumal College,Virudhunagar, in 1995 and MCA degree in Thiagarajar College of Engineering,Madurai and M.Phil (Computer Science) in Manonmaniam Sundaranar University,Tirunelveli. She has 15 years of teaching experience and she is currently working as Assistant Professor (Computer science) in Agricultural College an Research Institute, Madurai, TamilNadu, India. Her current research interest includes Image Processing, Wavelet, Curvelet and Neural Network. 